Available online @ https://jiem.jnnce.ac.in https:www.doi.org/10.37314/JJEM.2020.040105 Indexed in International Scientific Indiexing (ISI) Impact factor: 1.025 for 2018-19 Published on: 30 November 2020

\title{
An Enhanced Multi-Hop Routing Protocol for Wireless Body Area Networks
}

\author{
Sobiya Suha M Ahmed, Sunil M D \\ Department of ECE, J N N College of Engineering, Shivamogga \\ sobiyasuha@gmail.com,mdsunil.dev@gmail.com
}

\begin{abstract}
Routing is one of the main issues in designing wireless body area networks. Some drawbacks are revealed in existing routing technologies for practical networks: First, defective criteria e.g., only energy or distance is considered to elect the forwarder nodes. Second, the protocol's controllable parameters are decided manually, and for all application they stay fix, no automatic tuning is used. Third, the protocol is not altered and based on application specifications they remain optimized.

In order to get the better of the above mentioned drawbacks, an EMRP named protocol which is an adaptive Enhanced Multi-hop Routing Protocol is proposed and implemented in this paper. In this, the forwarder nodes are elected based on high energy, low communication distance and low path-loss and low energy consumption loss. The EMRP protocol's controllable parameters can be adaptively adjusted and tuned according to application requirement via genetic algorithm. With this view the paper focuses on study and implementation of protocols in NS2 such that it elects forwarder nodes with high energy, low communication distance, low path-loss and low energy consumption.
\end{abstract}

The implementation of (EMRP):Enhanced Multi-hop Routing Protocol and M-Attempt in NS2 and comparison of EMRP and M-Attempt protocols depending on various parameters like Packet-Drop, Energy Consumption, Packet Delivery Ratio (PDR) and Throughput. Network simulation results of the proposed protocol exhibit significant improvement compared to existing protocols in terms of lifetime, path-loss, PDR, energy consumption and throughput.

Keywords: Routing, implementation, Energy Consumption, PDR

\section{INTRODUCTION}

Wireless Sensor Networks (WSNs) are made of self-governing tiny sensors deployed randomly on a stipulated area. They collectively monitor existing environmental conditions like temperature, humidity, sound, strain, light, vibration and many other parameters. Monitoringtiny sized sensors sense and send accumulated data to base station /sink [3]. WSNs find applications in army, agriculture, domestic networks, medical field, and so on [4]. Latest developments in the field of electronics permit inserting of bio-sensors into the human body. Such a network is called as Wireless Body Area Network (WBAN). In WBAN, the sensor nodes forward the transmitted data to hospitals or to the sink for further examining. To deploy and to maintain the wired connection is highly costly. But, alternatively wireless connection is less costly and used comfortably in many applications. Moreover, with the wireless interface, the patient is not forced to remain in health center and is enabled with greater mobility [1] \& [5].

WBAN was introduced in 2001 by Van Dam et al. firstly [6]. Later, it wooed the 
attention of various research scholars [7]. The WBAN contains a group of tiny-size and less-power operated smart sensors that are implanted on human physique. The sensor nodes in WBANs have ability to establish wireless communication link with the sink. Thus it is used in real-time monitoring of health and helps in forwarding alarms and feedbacks to a server or health center [8]. When some sensors nodes in WBANs cannot directly send their information to its Sink, they depend on the forwarder nodes for their assistance to transfer their information to its sink, then a protocol for routing is employed. Free-space WSNs has monitored surrounding environment and it varies from WBANs. The network in WBANs is designed inside the human body ( $\mathrm{m}$ or $\mathrm{cm}$ ) and it considers few physical situations. If the nodes are damaged in WBANs their replacement is quite tough and also requires surgery for nodes that are implanted. Hence the network lifetime is prolonged in WBANs by avoiding replacement of nodes. In WBANs the sensors should not damage the human body, should utilize less energy required for data transmission and favours different body movements and they require more reliability as they monitor medical data. These traits of the human body need to be considered effectively, for proper functioning of the routing protocols in WBANs [7].

In this context, the paper presents the study and implementation of protocol in NS2 such that it elects forwarder nodes based on high energy level, and with less delay, path-loss, communicating distance and energy consumption parameters. The implementation of both protocols. The EMRP and M-attempt are implemented in NS2 and compared with various parameters like Packet Drop, Energy Consumption, PDR and Throughput.

\section{Literature review}

a) The authors in [10] has initiated an EMRP multi-hop protocol for WBANs. This includes problems of an existing techniques also explains defective criteria and to overcome these drawbacks, EMRP protocol is proposed in this paper and it considers the PDR, distance form sink, estimated consumption of energy and path loss of all the candidate sensor nodes for electing the forwarder parent nodes and proves excellency of EMRP protocol, through various experiments, by increasing the lifetime, throughput and decreasing energy consumption, path-loss is presented.

b) The authors in [2] has proposed a survey IEEE paper on WBANs. This paper contains review about developing research issues in WBANs like system's architecture, address allocating, routing, Physical layer, MAC layer, security issues and challenges and their applications is discussed. Moreover, it includes existent LEACH protocol in which every $\mathrm{CH}$ accepts packets of data from its each individual nodes, then $\mathrm{CH}$ transfers, the collected packets of data to sink which in turn decreases the direct transmissions number between individual nodes and its sink. These methods produces excess of overhead, congestion and large delay, during the process of clustering and selection of $\mathrm{CHs}$ is the main drawback which is discussed in this paper.

c) The authors in [11] has proposed a paper self-organization protocol named Anybody for WBANs. It involves the setup phase which includes formation of clusters by gathering the nodes, inter-connecting into clusters, it shows fixed number of the clusters are formed, when number of nodes is high \& finally establishing the suitable routing paths. Here every node behaves and adjust itself to its surrounding environment suitably in distributed manner. This kind of behaviour makes more adaptive, robust and scalable and extra overhead and delay problem is solved in this. Moreover, reliability and efficiency of energy are not explained effectively.

d) The authors in [12] has proposed energy efficient routing $\mathrm{M}$-attempt protocol for the WBANs. The paper contains a routing protocol which favours the human body mobility and it 
is a thermal-aware, that usually senses hot-links \& later routes, packets of data far away from the particular hottest links \& it uses TDMA scheduling for transmission between individual root node and sink for delivering packets of data to the sink. Here protocol chooses the path depending on the node which has low energy dissipation and lower hop count is presented in the paper) The author in [7] has proposed a paper on EECBR, an even or uniform energy consuming protocol, which helps in routing the nodes implanted behind the body, it is an enhanced effective data transmission protocol in WBANs. This paper explains energy efficiencies of the candidate nodes used to balance \& distribute the dissipation of energy amongst its sensors and depending on lowest standard deviation value candidate node is elected. The protocol also satisfies lifetime expansion, energy efficiency parameters of sensor nodes situated behind the human body is discussed.

f) The authors in [16] of has proposed a paper on simulation, analyzing of LEACH Protocol for WSNs using NS2 tool. This paper contains the work flow and work attributes of LEACH for WSNs using NS2 tool, and explains optimum number of $\mathrm{CHs}$ can decrease the energy consumption, and thereby increases the network performance. It also includes network scenarios, simulation results, analysis and methodology is presented in this paper.

\subsection{Communication model}

The three communication models are discussed below they are Network, path-loss and Energy consumption model. In this paper the network model is designed based on criteria like less path-loss of signal and less energy consumption in sending $\mathrm{n}$ - bit of data in the network, which results in prolonging Network's lifetime.

\subsubsection{Network model}

In WBANs, the data transmission may be either single-hop mode of transmission or two-hop is permitted, as stated by IEEE 802.15.6 [9]. Thus, with highest of two hop communication is considered in multi-hop routing. In most of the already existing protocol approaches, there exist no connections for the nodes placed behind or besides the human body. But, in this paper, it considers the sensor nodes placed on both behind, besides the body along with the nodes that are positioned on front side of the human body. Network model is expressed in the Figure 1.

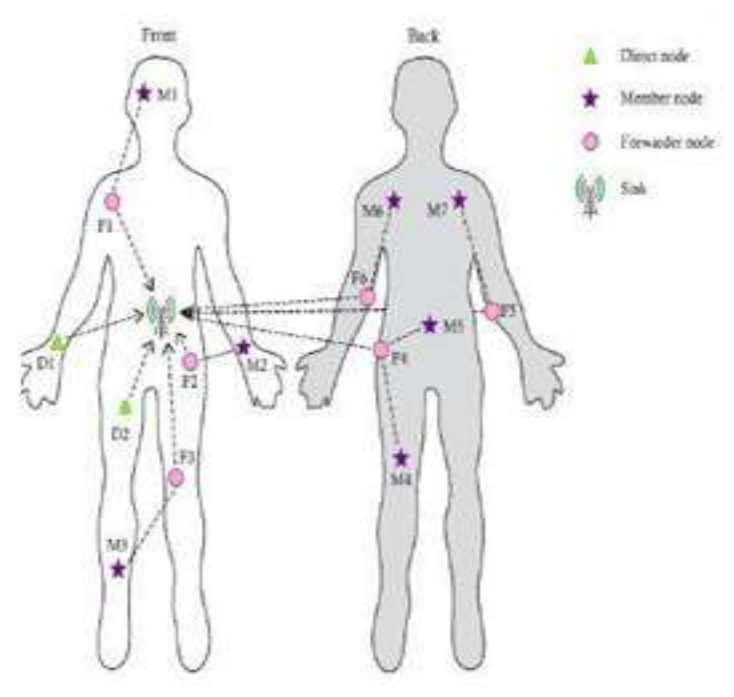

Figure 1: Network model

In order to sense data of the patient relating to ECG level, temperature, blood pressure, blood glucose level, \& so on, all the sensors are positioned on different parts of human's body and sink is situated at waist. In Figure 1, M4, M5, M6 are sensor nodes and M6 node is placed at backside of the body, for nodes placed at back it is difficult for directly transmitting the data packets to sink. As the single-hop transmission possess connection probabilities low and high path loss than multi-hop transmission via forwarder node. To sense its own location \& destination node's location, each node is provided with GPS and transmissions are through wireless interface. Here all sensors have an ability of transmitting with other sensor nodes and along with sink node. The operation of network is separately classified into rounds. Every node is able to 
sense its data \& transmits it directly through a relay node to the sink, at each round.

In Figure 1, all sensor nodes are classified into three categories: a) Direct node represented by $\mathrm{Di}$, that directly sends its data to the sink, b) Member node represented by Mi., that transmits its data via parent / forwarder node, c) Forwarder node represented by $\mathrm{Fi}$, that aggregates all its data from all member nodes and sends it along with its data to sink. In two consecutive time intervals, data transmission is achieved in every round. In first time period, every member node transmits its data packet to its respective forwarder node. Later, all the direct and relay nodes sends their own data packets to sink in the second time period.

\subsubsection{Path-loss model}

Path-loss is reduction in power attenuation and it is stated as difference of the power transmitted and the received power at the destination node of the signal. It is the signal's attenuation that are calculated in decibels (db) [14]. But in WBANs, because of motion of body parts and various postures of body, the signal may get damaged. Path-loss is a relation of distance (D) and frequency (F) among transmitter and receiver and is given by

$$
P L(d b)=P L_{o}+10 \eta \log _{10}\left(D / D_{o}\right)+A \sigma
$$

Where, $\mathrm{D}_{0}$ - reference distance, $A \sigma$ Random Gaussian variable having mean 0 , as well as standard deviation of $\sigma_{\eta}$ - path loss exponent. The received powerP $P L_{o}$ at $\mathrm{D}_{0}$, can be expressed as Eq. (1), c is constant speed of the light.

$$
P L_{o}=20 \log _{10}\left(\frac{4 \pi D_{o} F}{c}\right)
$$

Path-loss of WBANs is much high as compared with free-space WSNs, because in WBANs communication is through human body [17]. The path-loss value for WSNs is 2, also for WBANs, it differs in between three to four for a LOS \& five to seven for a NLOS[7]

The $\mathrm{D}_{0}$ value is $10 \mathrm{~cm}$. It is tough to accurately estimate power of a signal, across transmitter node \& receiver node. So, the Gaussian variable ' $A \sigma$ ' is added in Eq. (1).

\subsubsection{Energy consumption model}

The two radio transmission models are considered in this paper. For transferring an n-bit of data packets, with distance $d$ between them. And consumption of energy, in a transmitter node $\left(E_{T}\right) \&$ in a receiver node $\left(E_{R}\right)$ can be respectively represented in Eq. (3) and (4) [7].

$E_{T}(n ; d)=E_{\text {T-elect }} \times n+E_{\text {amp }} \times n \times d^{n}$

$E_{R}(n ; d)=E_{R \text {-elect }} \times n$

\section{Methodology}

In proposed methodology of this paper, as the sensors are implanted on human body to sense various health conditions like temperature, sugar level, blood pressure etc. This sensor node monitors and transmits data packets to sink or medical cares for further examining. The initial level is the ground level which contains.

Sensor nodes and the application level is health center. In this proposed paper we are working between these two levels. In paper, the Figure 2 explains, the Methodology of proposed work.

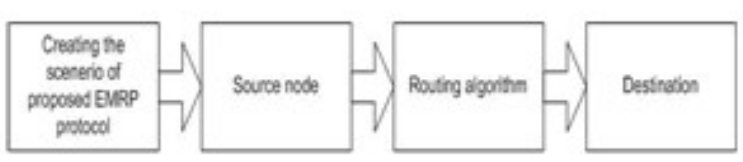

Figure 2: Methodology of Proposed work

As we are concentrating on network side not on the genetic side. Initially we are creating the scenario of EMRP protocol in NS2. The processed output from Genetic algorithm is process through the network i.e., without losing information and without 
dropping of any packets are conveyed to the same network and with the encryption providing security to the network and if any malicious or packet drop occurs means rectifying it and recover the message using routing algorithm and transferring it to the destination or sink.

\section{Proposed EMRP protocol}

The multi-hop protocol called EMRP is presented in this paper. EMRP is multi-hop routing, flexible and application-specific protocol for WBANs. Communication is direct transmission (single-hop) or transmission through relay node (multi-hop). Residual forwarder energy, distance, energy consumption, position of node \& packet loss are considered in electing a suitable forwarder node to transmit data that is sensed, of each member node to the sink. According to application specification the parameters of routing EMRP protocol are automatically adjusted using genetic algorithm (GA). GA is guided by multi-objective fitness function which tunes the EMRP parameters according to application specification.

\subsection{Operation of Proposed EMRP protocol}

The operations in EMRP protocol for every round is categorized into 2 phases i.e., setup and steady-state phase. In setup phase the EMRP functions for electing a suitable Routes for each node. Hence accordingly to the multi-objective function proposed, the communication selected is either one-hop communication or two-hop for each of the node. If single-hop routing has improved performance, then that node is decided as the direct node. If node contains one of the member node, then it is two-hop, else it is direct node or single-hop communication. In this case, it decreases the problem of routing by electing the forwarder node among these single-hop nodes. And all computations for routing procedure are handled in the sink i.e., the sink, gathers data packets about energy level \& current location of all the nodes at starting of each round. So, the sink has the knowledge about current location and remaining energy of every nodes in each of the round.

When all routing paths are performed, a sink node establishes the time scheduling operation by TDMA to decide at what time the direct node \& forwarder sensor nodes can send their data to the sink. Later, sink sends the advertising message which includes the outcomes of routing protocol. Each forwarder node sends an advertisement for its member nodes, that it is a relay to all of them at current round, and tell them when they need to interact with a relay node. In steady phase, here each of the member node transmit their data packet to its own forwarder node in fixed time intervals in scheduling one. Later, all direct node \& forwarder nodes transmit their own data packets to the sink in scheduling two. Here every forwarder node transmits data packets of its member nodes along with its data packets, while in direct communication each node transmits only its data to the sink.

\subsection{Direct or multi-hop communication selection strategy}

As discussed, the communication in EMRP allowed is only single-hop transmission through direct and forwarder nodes or multi-hop transmission through member nodes. So, the routing problems may be reduced into 2 steps respectively. In step one, EMRP decides that transmission of each sensor nodes can be either single-hop Transmission or a two-hop, and later in the next step, a suitable forwarder node must be elected for every member node in multi-hop transmission. And all other nodes are decided as forwarder node for each study node. In EMRP protocol, each node which proves all conditions of Eq. (5) (6) and (7), is summed with the troop of all candidate nodes, to be the relay node of a study node.

$p l(x) \leq \alpha \times p l($ Direct $)$,

Where $x=1,2,3, \ldots \mathrm{N}, x \neq$ study node

$e c(x) \leq \beta \times e c($ Direct $)$, 
Where $x=1,2,3, \ldots \mathrm{N}, x \neq$ study node $r e(x) \geq \delta \times \frac{1}{N} \sum_{y=1}^{N} r e(y)$.

Where $x=1,2,3, \ldots \mathrm{N}, x \neq$ study node

Where, $\mathrm{N}$ - Number of the alive nodes $p l(x)=p l_{1}(x)+p l_{2}(x) \& \quad e c(x)=e c_{I}(x)+$ $e c_{2}(x)$

In Eq. (5), $p l_{1}(x)$ - path-loss from a study node to forwarder node of first link, $p l_{2}(x)$. Path-loss from a relay node to sink node of second link \& pl(Direct) - path loss from a study node to sink which is direct transmission. In $(6), e c_{1}(x), e c_{2}(x)$ and $e c($ Direct) are dissipated total energies of network, because of transmission through first link, second link \& direct transmissions respectively. Eq. (7), $r e(x)$ - residual energy level of a node. Also, parameters $\alpha . \beta \& \delta$ are used for tuning the selection model among the single-hop or the two-hop transmission.

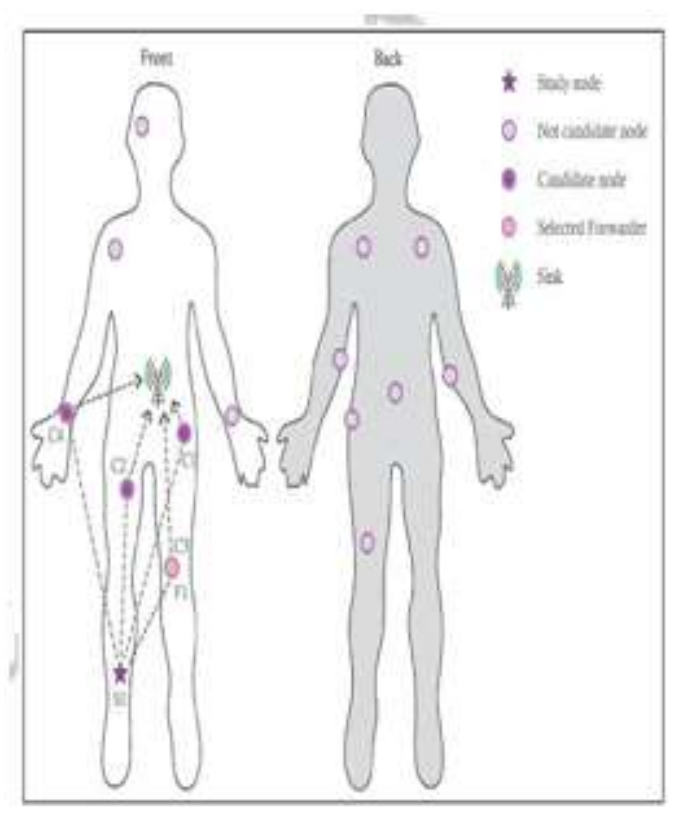

Figure 3: Selection strategy of forwarder node in EMRP

If it contains empty set of the candidates, then study node is one-hop node. Else, study node is two-hop transmission. This procedure of EMRP is demonstrated in the Figure 3. In the figure there are 14 nodes placed at front and backside / behind the body, S1 is the study node and 4 nodes- $\mathrm{C} 1, \mathrm{C} 2, \mathrm{C} 3$ \& $\mathrm{C} 4$ are candidate nodes. If the candidate nodes satisfies the two conditions of eqn. (5), (6), (7),then that study node is decided to be two-hop. Later among 4 candidate nodes, one node in four nodes is decided as forwarder node.

In accordance with the objective function proposed, C3- candidate node has been elected finally as the parent forwarder of S1- study node. While C4 is study node, from all other 14 nodes if two conditions of eq. are not satisfied means. Then the node $\mathrm{C} 4$ is pointed as the one-hop node.

\subsection{Procedure of selecting the parent node}

To elect the parent forwarder for each member node, EMRP uses residual energy, its distance from the study sensors to sink, estimated path loss \& energy consumption. EMRP elects the suitable parent node which has high level of energy $\left(F_{1}\right)$, low istance $\left(F_{2}\right)$, low energy consumption network $\left(F_{3}\right) \&$ low path $\operatorname{loss}\left(F_{4}\right)$. Multi-objective function is proposed for electing a candidate node $x$ which is defined as

$$
\begin{aligned}
& O F(x)=W_{1} F_{1}(x)+W_{2} F_{2}(x)+W_{3} F_{3}(x)+ \\
& W_{4} F_{4}(x), \forall x \in C N
\end{aligned}
$$

Where, $\quad W_{1}, W_{2}, W_{3}, W_{4}-\quad$ weighting parameters. $\mathrm{CN}$ - set of candidate nodes.

$\left(W_{1}+W_{2}+W_{3}+W_{4}=1\right)$ to tune the importance of the four objectives $F_{1}$ to $F_{4}$. The four parameters of objective function for each of the candidate node, which is expressed

Accordingly in Eq. (9) to (12).

$$
\begin{aligned}
& F_{1}(x)=\frac{r e(x)^{-1}}{\sum_{y=C}\left(r e(y)^{-1}\right)} \\
& F^{2(x)}=\frac{d^{1(x)}+d^{2}(x)}{\sum_{y=C N}\left(d^{1}(y)+d^{2(y)}\right)} \\
& F^{3(x)}=\frac{e c^{1(x)}+e c^{2}(x)}{\sum_{y=C N}\left(e c^{1}(y)+e c^{2(y)}\right)} \\
& F^{4(x)}=\frac{p l^{1(x)}+p l^{2}(x)}{\sum_{y=C N}\left(p l^{1}(y)+p l^{2(y)}\right)}
\end{aligned}
$$


First objective in eqn.(9) is residual energy parameter which balances the uniform dissipation of energy among all its sensors. If any node has high energy level then it has higher chances to be selected as the forwarder node. And the Eq. (10), is distance between a study node sensors to candidate node(first link's distance), is distance from a candidate node to sink node (second link's distance). Parameters in Eq. (10) and (12) is distance \& the path loss which provides successfully delivering of the packets to sink. And in eqn. (11) is energy consumption which minimizes the overall energy dissipation of a network. And when the objective function is calculated for every candidate nodes, a node with least quantity of objective function is elected to be the forwarder of that study node. The type of operation is carried out for every member nodes, which create all its routes in the network

\subsection{EMRP protocol optimization via Genetic algorithm/ GA}

In WSNs it has various application specific clustering protocols, which adjust its parameters automatically depending on application requirement. But, EMRP routing technique is first multi-hop application precise protocol for WBANs. Usually, in existing routing model the controllable parameters are decided manually, and they stay fix for all the approaches. The EMPR's controllable Parameters are $\alpha, \beta, \delta, W_{1}, W_{2}, W_{3}, W_{4}$. It is very important for properly electing these parameters. So, to fix the parameters of protocol automatically according to application is performed through optimization technique.

The optimization technique of EMRP is handled with optimization algorithms as a heuristic, evolutionary \& swarm intelligent algorithms. GA is chosen in this paper, as it is mainly easy and good overall \& local-search capacity. Genetic Algorithm is an improved routing algorithm, established in 1975 by Holland [18]. In this GA, the random initial population is created and it has two steps population evaluation and population updating are consecutively carried out, till it reaches its particular iterations count.

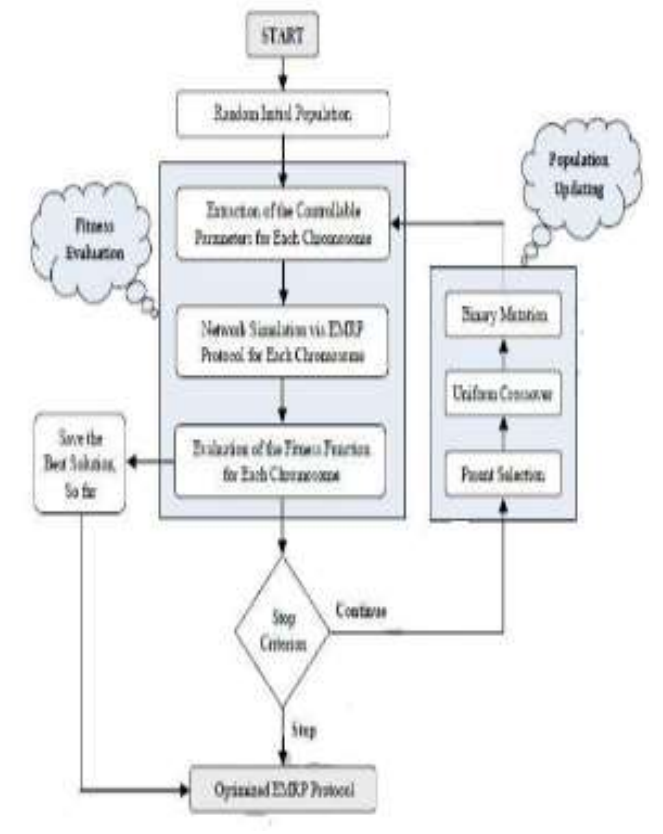

Figure 4: Flowchart of Genetic algorithm optimization technique for tuning EMRP routing protocol

The fitness function may be evaluated depended on the network performances like lifetime, drop, delay, PDR \& throughput or other parameters accordingly to the various approach specifications. The complete flowchart of optimization technique shown in Figure 4. Stop criterion of GA method is termination of specified count of the iterations. The GA algorithm is stopped, when it reaches particular number of the iterations and the overall best solution is obtained. This is a final solution of an algorithm.

\begin{tabular}{|l|l|l|l|l|l|l|}
\hline $\mathbf{W}_{\mathrm{V}}$ & $\mathbf{W}_{2}$ & $\mathrm{~W}_{3}$ & $\mathbf{W}_{i}$ & $\alpha$ & $\beta$ & $\delta$ \\
\hline
\end{tabular}

Figure 5: Chromosome representation suitable solutions for tuning the parameters of EMRP.

As discussed, the controllable parameters are $\alpha, \beta, \delta, W_{1}, W_{2}, W_{3}, W_{4}$. Figure 5 is Chromosome representation an suitable solution for tuning parameters of the EMRP. The EMRP routing is flexible protocol and any change in parameters valves affects, the EMRP operation. Hence the parameters are designed 
according to the required characteristics of the population of an algorithm is generated randomly and constant random number is produced for each of the gene, not beyond its pre-determined value of that gene and hence random solution is constructed.

\subsection{Fitness evaluation and Updating of population in GA}

Fitness function is defined depending on one or more performances of the network like delay, throughput, drop, network lifetime \& various different criterions based on different characteristics of application. To estimate the solution, chromosome is then decoded to get best result of controllable parameters of the proposed protocol.

Table 1: Limitations in M-Attempt protocol \& solutions through the proposed EMRP

\begin{tabular}{|c|c|c|}
\hline Funtions & Linitstiorsin MAttempt putacol & Solutiontragen EMAP putocd \\
\hline 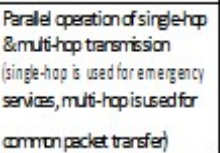 & $\begin{array}{l}\text { Nocorsideration of the rodes } \\
\text { that are siturted at bedk ff the } \\
\text { bod/(if sinkrode is prest on the } \\
\text { frotside) }\end{array}$ & 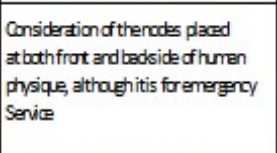 \\
\hline $\begin{array}{l}\text { Energy consumption and extarion } \\
\text { of thentwork Ifetine }\end{array}$ & $\begin{array}{l}\text { Selection of arates that hes a } \\
\text { lover hop ourt inthemiti-hop } \\
\text { Commuicstion }\end{array}$ & $\begin{array}{l}\text { Considation d cursumption } \\
\text { energy\&:residal enegy of } \\
\text { all sersors }\end{array}$ \\
\hline Pustural charge curtrol & 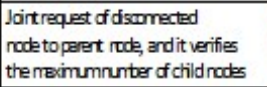 & $\begin{array}{l}\text { Consideraion f thepricities } f \text { the } \\
\text { child node w hen it reaches the maximum } \\
\text { Defege }\end{array}$ \\
\hline $\begin{array}{l}\text { Paranaters for selecting forvarder } \\
\text { Nccte }\end{array}$ & 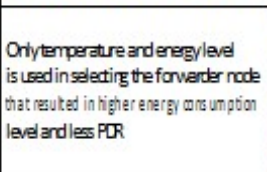 & 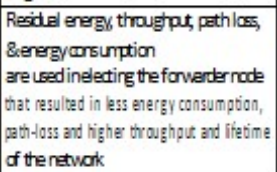 \\
\hline
\end{tabular}

Population updating is differentiated into 3 parts i.e., reproduction, mutation $\&$ crossover. In every iteration, the percent of best population with maximal objective function is shifted to next iteration. In, uniform crossover presented in Figure 6, every gene of an offspring from one of the parents is shifted randomly. And in mutation, a gene chosen is the random one, and later its value are changed randomly, within pre-determined value of that gene. Mutation elects chromosome of high performance via Fitness selection called as roulette wheel. It is a value-based mutation operation shown in Figure 7.

\subsection{Limitations of M-Attempt protocol \& solutions through the proposed EMRP protocol}

While analyzing the disadvantages of M-ATTEMPT protocol, a new protocol is proposed as solution protocol to an existing. Table 1 outlines the major limitations in M-ATTEMPT protocol \& solutions through the proposed EMRP protocol.

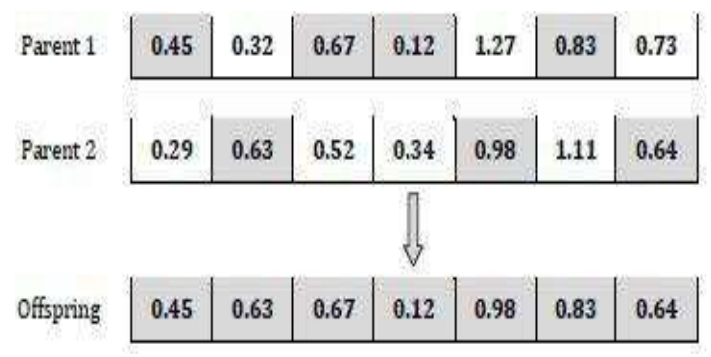

Figure 6: Crossover operation

\section{Implementation steps of protocols in} NS-2

All simulations are implemented and executed in NS2. The proposed routing EMRP protocol for WBANs is distinguished against a M-ATTEMPT protocol, the energy \& temperature aware protocol. As in NS2 tool TCL scripts are used for simulation. Features required for writing TCL scripts is mentioned below.

a. Creating wireless nodes scenarios, While creating the scenario we require to describe parameters like antenna types, radio frequency propagation model, routing protocol type used by nodes that are mobile, type of queue etc.

b. Creating node-connections that are either simplex / duplex links or LANs.

c. Creating topologies for the network that is either Grid-based, Random, Chain topology.

d. Creating agents TCP, UDP. And it also supports the TCPSink, TCP/FullTCP, TCP/Reno, TCP/New-Reno, Null agent and $\mathrm{TCP} /$ Vegas.

e. It supports DSDV protocol, AODV protocol, DSR \& TORA protocol for routing mechanism. 
f. It supports applications FTP and CBR, Telnet, Http/server, Http/client, Http/cache, Web traf, Traffic /CBR, Traffic/Pareto, Traffic /Exponential.

g. It supports node mobility in the network.

h. Setting the size of packets, start time and end time for simulation process, transmission ranges, bandwidth and interference range in wireless networks, etc

\subsection{Settings for simulation}

To prove excellence of proposed EMRP protocol, different experiments in NS2 are performed. The simulating area of size $0.9 \mathrm{~m} \times$ $1.7 \mathrm{~m} \times 0.4 \mathrm{~m}$ is considered.

Table 2: Details of Network

\begin{tabular}{|c|c|}
\hline Parameter & Specification / Value \\
\hline Tool for simulation & Network simulator version(ns- 2.35) \\
\hline IFEE Standards & IEEE 802.15 .6 \\
\hline Total number of the nodes & 35 \\
\hline Total number of $\mathrm{CH}$ & 4 \\
\hline $\begin{array}{l}\text { The size of network } \\
\text { (in m or } \mathrm{cm} \text { ) }\end{array}$ & $0.9 \mathrm{~m} \times 1.7 \mathrm{~m} \times 0.4 \mathrm{~m})$ \\
\hline Node Range & $18 \mathrm{~m}$ \\
\hline Sink & 1 \\
\hline Initial eae rgy of nodes & 1 joule \\
\hline$E_{T-\text { ded }}$ & 16,8 nJoule/bit \\
\hline$E_{R \text {-ded }}$ & 35.2 nJoula/bit \\
\hline$E_{\text {amp }}$ & $1.97 \mathrm{n} / \mathrm{bit} / \mathrm{m}$ \\
\hline$D_{0}$ & $12 \mathrm{~cm}$ \\
\hline $\mathrm{C}$ & $3 \times 10^{3}$ \\
\hline$\eta$ (for Line Of Sight) & 4 \\
\hline$\eta$ (for Non Line Of Sight) & 7 \\
\hline Freq & $2.5 \times 10^{2}$ Hertz \\
\hline $\mathrm{n}$ (size of the data packets) & 6000 bit \\
\hline
\end{tabular}

The network has 35 total numbers of nodes, 4-cluster heads, 1-sink node. Each sensor node has an initial energy of 1 joule. Here sink is positioned at middle of the human physique to efficiently receive maximum data packets from the sensors. Network's details \& simulation parameters is outlined in Table 2

\section{Network simulation results}

This section contains network simulation of nodes deployment $\&$ the comparative results of a EMRP protocol for Path-loss, Throughput,
Energy consumption and Packet Drop which was performed using NS-2 tool.

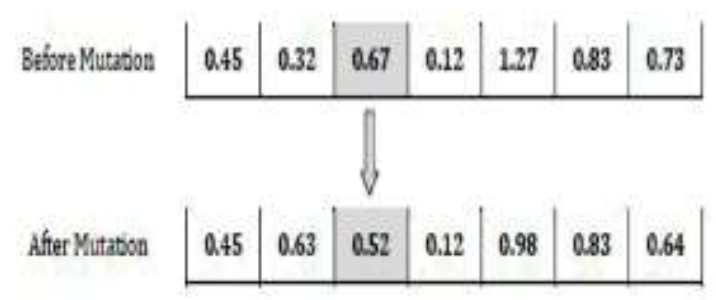

Figure 7: Value-based mutation operator

\subsection{Deployment of the Sensor nodes}

Simulation is performed with 35 nodes for both the protocols. Here the sensors deployment is in random fashion which is presented in Figure 8 . The network is established with 26 nodes and 4 distributed gateway node, 2 common gateway nodes, 1 base station and troop identification process is completed i.e., forming of member nodes, selection of the cluster head and sink which is represented in Figure 9. The Figure $10 \& 11$ represents the data transmission with packet drops by utilizing specific routing path and data packets moving to base stations respectively.

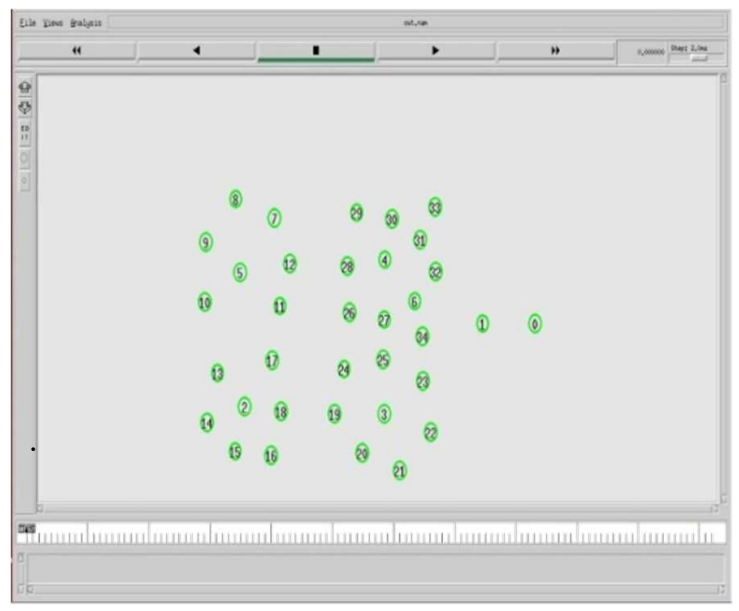

Figure 8: Random deployment of the sensor

\subsection{Comparison Results}

The comparison is performed for Energy consumption, Throughput, Packet drop and Packet delivery ratios between the EMRP and M-Attempt protocol. 


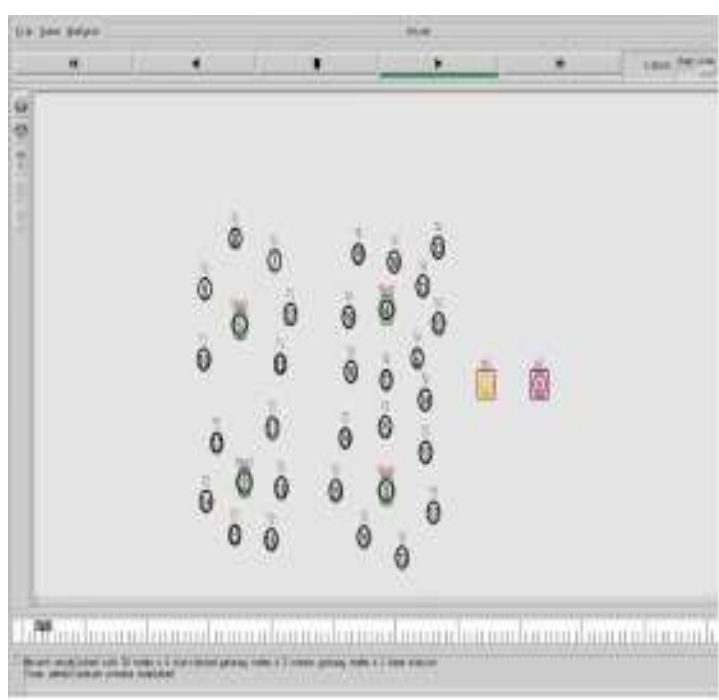

Figure 9: Formation of the troops

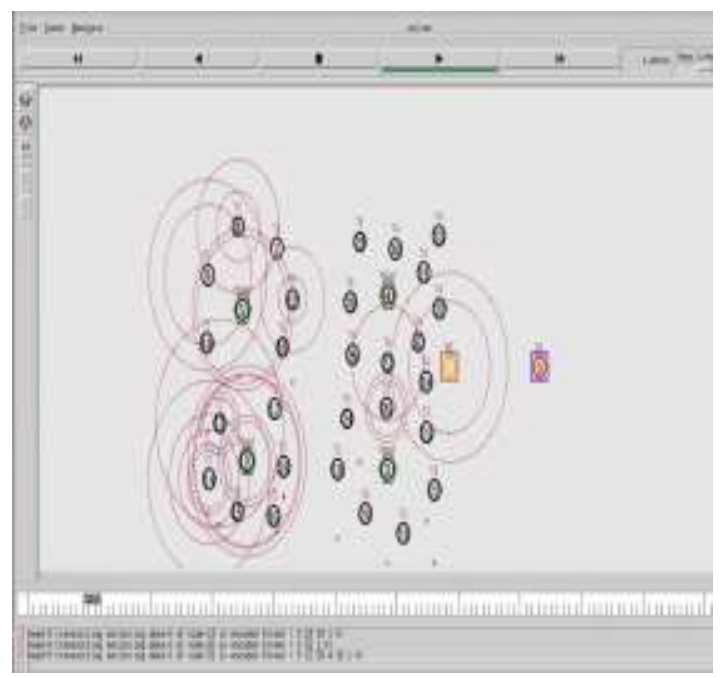

Figure 10: Data transmission from $\mathrm{CH}$ and its members to sink

\section{- Energy consumption:}

Figure 12 represents the comparison of residual energy of the network for Proposed EMRP and M-Attempt protocol. Due to potency \& efficiency of proposed EMRP routing for WBANs, all the sensors in EMRP consume less energy on an average. Thus, it saves more energy and lifetime of the network is prolonged. These results show that the EMRP protocol has more energy efficiency and less energy consumption than M-Attempt protocol.

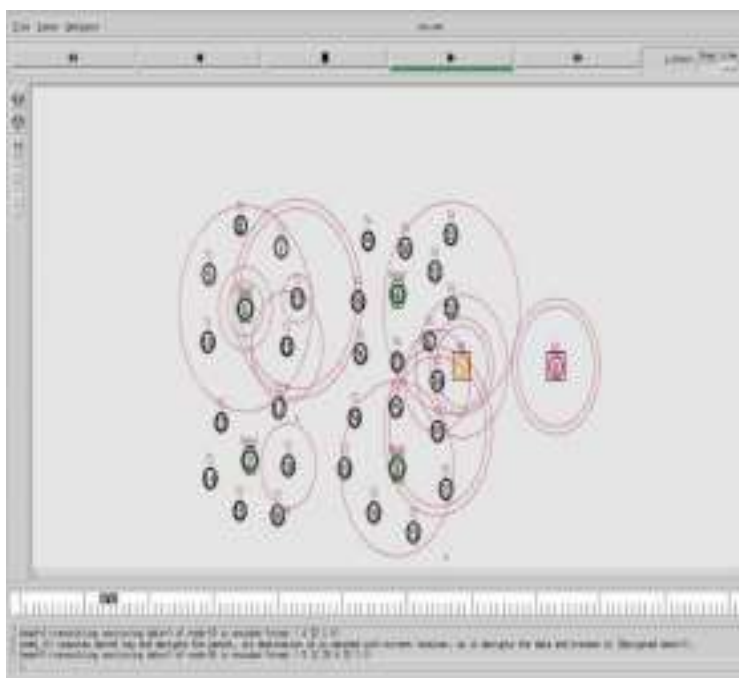

Figure 11: Data packets received by sink

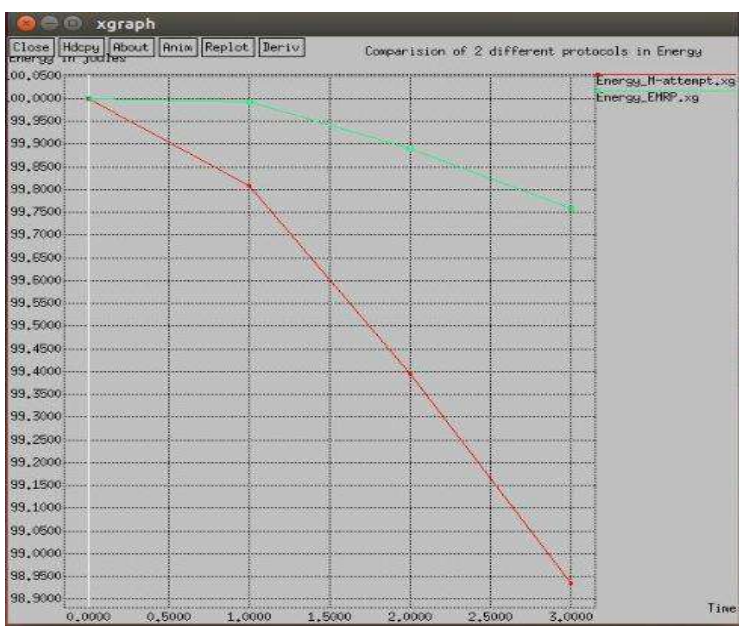

Figure 12: Comparison of 2 different protocols w.r.t Energy consumption

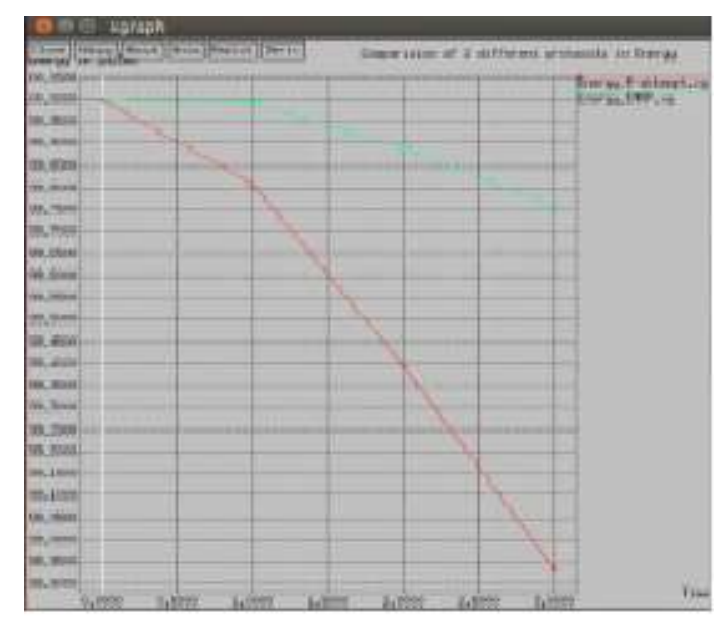

Figure 13: packet drop curve 


\section{- Packet drop:}

The packet-drop defines number of the packets sent to number of the packets received. From these Packet Drop's curve that is presented in Figure 13. And concluded that M-Attempt have more drop of packets than EMRP protocol.

\section{- Throughput:}

Number of successfully received data packets to the destination within specified period of time. From these Throughput curve i.e., shown in Figure 14. All nodes in EMRP protocol guarantees better in successfully transmitting data to the sink than M-Attempt protocol.

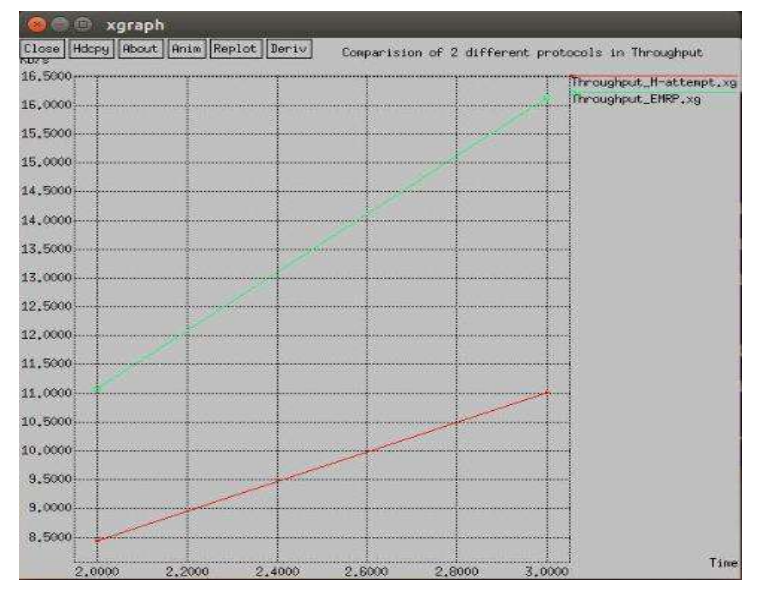

Figure 14: Comparison of 2 different protocols w.r.t Energy consumption

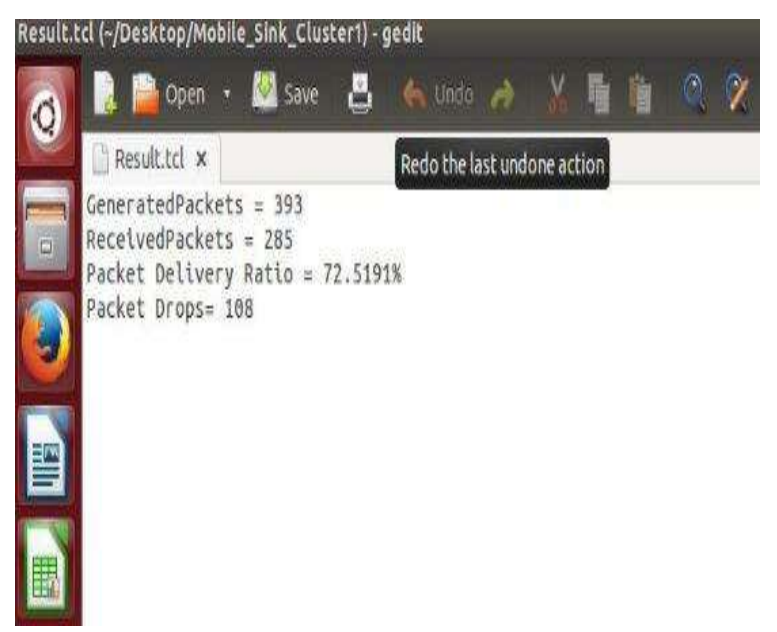

Figure 15: PDR and Packet drop is obtained for the designed network when simulated

\section{- Packet Delivery Ratio(PDR)}

PDR is depended on generated packets and received packets. And it is stated as, ratio between received packets by a sink / destination, to generated packets by a source. In Figure 15 the PDR of the network is 72.51

\section{Conclusion}

The existing techniques have the following limitations: 1. No consideration of nodes placed at back side of the body 2 . The parameters like energy \& temperature are used in selection of forwarder node 3. Existing protocols cannot be altered or tuned based on application requirements.

In order get solutions to these limitations of existing technique, EMRP considers the energy, the distance from sink, the approximated path- loss \& the energy efficiency of all candidates for electing the suitable forwarder nodes. The excellence of proposed routing EMRP protocol's methodology is verified through creating the wireless- Scenario in NS2. According to results that are obtained, it concludes EMRP protocol's performance is better than the existing M-Attempt technique in terms of increasing network's lifetime, throughput \& decreasing energy consumption, path-loss.

\section{References}

1. M. I. Latre B, Braem, "A surveyon wbans," Wireless Networks, vol. 17, no. 1.

2. S. Movassaghi, "Wireless body area networks: A survey," IEEE Communications Surveys \& Tutorials, vol. 16, no. 3.

3. C AkyildizI, Sankarasubramaniam, "Survey on wireless sensor networks," Computer networks, vol. 38, no. 4.

4. K. E. B.A Attea, "A new evolutionary based routing protocol for clustered heterogeneous wsns," Applied Soft Computing, vol. 12, no. 7.

5. G. Kumar, "On-body measurements of ss-uwb patch antenna for wban 
applications.," AEU-International Journal of Electronics and Communications, vol. 70 , no. 5 .

6. B. Van Dam, Pitchers S, "Body area networks towards a wearable future," In Proceedings of WWRF kick off meeting, Germany,.

7. Ha, "Even energy consumption and backside routing (eecbr) an improved routing for effective data transmission in wbans," International Journal of Distributed Sensor Networks, vol. 12, no. 7.

8. J. S. Park, "Enhancing the quality of life through wearable technologies," IEEE Engineering in Medicine and Biology Magazine, vol. 22, no. 3.

9. Latre, "Ieee p802.15.6/d0 draft standard for body area network," IEEE Draft.

10. B. Esmaeile, "Evolutionary-based multi-hop routing protocol for wireless body area networks(emrp)," International Journal of Electronics and Communications, June-2018.

11. D. Watteyne, Blum, "A self organization protocol for wbans (anybody)," 2nd International Conference on Body Area Networks, Florence, Italy,.
12. A. Javaid, Fareed, "A new energy efficient routing protocol for wireless body area sensor networks(m-attempt)," Procedia Computer Science,, vol. 19, no. 224-231.

13. L. Abolhasan, "Energy efficient thermal and power aware routing (etpa) in body area networks," In 23rd IEEE International Symp. on Personal Indoor and Mobile Radio Commun. (PIMRC).

14. J. Nadeem, "Stable increased through put multi hop protocol(simple) for link efficiency in wbans," In Eighth International Conference on Broadband and Wireless Computing, Communication and Applications (BWCCA).

15. B. Chandrakasan, "Energy efficient communication protocol for wireless microsensor networks," In Proc. Hawaii Int. Conf. on System Sciences.

16. Y. Tian, Du, "Simulation and analysis of leach protocol for wsns based on ns2," International Conference on System Science and Engineering, Dalian, China, vol. 28, July 2012.

17. B. Ullah S, "A comprehensive survey of wbans," J Med Syst, vol. 36, no.3.

18. Holland, "Adaptation in natural and artificial systems," in University of Michigan press, Ann Arbor, 1975. 